\title{
Clinicopathological Profile of Lung Cancers at an Institute from South India-A Record Based Retrospective Cohort Study
}

\author{
Vinod Ramani ${ }^{1}$, Choudhury Bijit ${ }^{2}$, Sarathy Vinu ${ }^{1}$, Jayappa Srinivas Belagutti ${ }^{1}$, Naik Radheshyam ${ }^{1}$ \\ ${ }^{1}$ HealthCare Global Enterprise Ltd., Bangalore, India \\ ${ }^{2}$ Biocon, Bangalore, India \\ Email: vinodramani77@gmail.com
}

How to cite this paper: Ramani, V., Bijit, C., Vinu, S., Belagutti, J.S. and Radheshyam, N. (2020) Clinicopathological Profile of Lung Cancers at an Institute from South India-A Record Based Retrospective Cohort Study. Advances in Lung Cancer, 9, 41-54.

https://doi.org/10.4236/alc.2020.93005

Received: September 2, 2020

Accepted: September 27, 2020

Published: September 30, 2020

Copyright $\odot 2020$ by author(s) and Scientific Research Publishing Inc. This work is licensed under the Creative Commons Attribution International License (CC BY 4.0).

http://creativecommons.org/licenses/by/4.0/

\begin{abstract}
Introduction: Apart from smoking as the known risk factor for lung cancer, recent developments implicate occupational exposure to carcinogens, indoor air pollution and dietary factors as other causative agents. In our study, we have analyzed the clinical and pathological profile of lung cancer patients treated at our center over a period of 8 years. Aim: To find the demographic and clinicopathological profile of lung cancer patients admitted to the oncology unit. Methods: This retrospective record based analysis includes a cohort of 1248 patients diagnosed with lung cancer, at a tertiary cancer care center in Bangalore, South India. This study includes data of patients admitted during the period 2010 to 2018, retrieved from the Hospital's Electronic Medical Records (EMR). Their demographic profile, clinical correlates, radiological profile and diagnostic details were studied. Benign tumours, malignant pleural disease and sarcomatoid tumors were excluded from this study. Results: Adenocarcinoma (AC) was detected among $70.4 \%$ of patients, Squamous cell carcinoma (SCC) among $15.3 \%$ and Small cell lung cancer (SCLC) among $14.3 \%$ of lung cancer admissions. Male to female ratio was 2.95:1. It was found that the median age of lung cancer patients was 61 years. $>60 \%$ of lung cancer patients were from the 51 - 70 year age-group. Across the three types of cancers among the patients in Stage 3B to 4 strata, $>35 \%$ received palliative chemotherapy and $>20 \%$ received palliative chemotherapy + radiation. Conclusion: In our study, AC is the most common histological subtype (>70\%) of lung cancer. The outcome of lung cancer patients can be considerably impacted by addressing risk factors through preventive measures implemented in the community.
\end{abstract}

\section{Keywords}

Lung Neoplasms, Adenocarcinoma, Squamous Cell Carcinoma, Small Cell 


\section{Introduction}

GLOBOCAN 2018 reports that globally, lung cancer contributes to $11.16 \%$ of all cancer cases and $18.4 \%$ of all cancer related deaths. In India, its incidence per 100,000 is 7.8 among males and 3 in females [1]. Noronha $V$ et al. [2] mention that the adenocarcinoma (AC) trend among lung cancer cases across the globe is paralleled by that in India. However, we need to assess the rise in incidence of lung cancer among non-smokers. Small cell lung cancer (SCLC) is presumed to constitute $14 \%$ of all lung cancers [3].

Apart from smoking as the known risk factor for lung cancer, recent developments implicate occupational exposure to carcinogens, indoor air pollution and dietary factors as other causative agents. Vegetable and fruit intake are protective factors for lung cancer, whereas animal food and dairy products are known to have predisposing effect on the cancer [4]. Urban air pollutant including indoor air pollution is a known risk factor for lung cancer. The role of oxidant/antioxidant imbalance in the pathogenesis of lung cancer is also documented. In the Western Countries, women are being increasingly diagnosed with lung cancer, and $\mathrm{AC}$ is the most common histological cell type ahead of Squamous cell carcinoma (SCC) [5]. Jindal S.K [6] opined that the cell type of cancer is largely influenced by characteristics such as age, sex and smoking habits. SCC tends to exclusively occur among smokers and in males. In India, a larger proportion of smokers are males and thus females will have an inverse increase in AC. Age also influences the cell type pattern, where-in SCLC tends to occur among $<40$ year old individuals irrespective of their smoking status. However among those $>40$ years, SCC is common among smokers and AC among non-smokers [6]. Rawat J et al. [5] report the delay in seeking treatment in the range of 4 to 6 months.

In India, due to the wide prevalence of tuberculosis, many lung cancer patients could be initially treated otherwise. However, lung cancer diagnosis is favored given the age of the patient, history of smoking, signs of superior vena cava obstruction, mediastinal symptoms such as hoarseness of voice and dysphagia. On examination, there could be signs of collapse or mass, clubbing of nailbed and other complications of lung cancer (both metastatic and non-metastatic). The commonest radiological finding in lung cancer includes mass with or without collapse [4]. Among the common histological sub-types, presentation of mass in the lung is either central or peripheral: Adeno $(38.3 \%, 61 \%)$, Squamous $(72.2 \%$, $27.8 \%)$ and Small cell $(83.6 \%, 16.4 \%)$ respectively. Cytopathological examination enables diagnosis and categorization of cell type for initiating the line of management.

Chromosomal changes in lung cancer such as numerical abnormalities and 
structural aberrations (including deletions and translocations) have been identified through cytogenetic studies. Small cell lung cancer is associated with activation of the dominant cellular protooncogenes like c-myc, L-myc, N-myc, c-raf and inactivation of the recessive or tumour suppressor genes like p53 and Rb. Non-small cell lung cancer is associated with K-ras, N-ras, H-ras, c-myc, c-raf and tumour suppressor genes like p16 and Rb genes [7]. Apoptosis is altered in lung cancer due to changes in anti-(BCL-3, Bel-xl) and proapoptotic members (Bax, Bad). The tumour suppressor gene FHIT is frequently altered in lung cancer [4].

In India, data regarding site wise distribution of cancer can be gathered from population based cancer registries. However, such data do not report the histological subtypes [8]. Thus, it is imperative to analyze data from hospital based registries. In our study, we have analyzed the clinical and pathological profile of lung cancer patients treated at our center over a period of 8 years.

Aim: To find the demographic and clinicopathological profile of lung cancer patients admitted to the oncology unit.

\section{Material and Methods}

This retrospective record based analysis includes a cohort of 1248 patients diagnosed with lung cancer, at a tertiary cancer care center in Bangalore, South India. This study includes data of patients admitted during the period 2010 to 2018, retrieved from the Hospital's Electronic Medical Records (EMR). This hospital from its centers located across India, provides comprehensive cancer care services for Indians as well as foreign citizens.

Healthcare Global (HCG) is the largest private cancer care provider in India. It has a network of 27 comprehensive cancer centers in India and abroad. Each center is provided with a business system, management expertise and capital resources to bring patient focused, state of the art cancer care. The hub and spoke model has helped to create an integrated approach to cancer care. $>120,000$ patients are treated through our centers annually. Also, the HCG foundation enables need-based patients through concessions and waiver of hospitalization costs. Since the foundation's inception during December 2006, around 1300 financially deserving patients have derived benefit from this noble cause.

Patients with confirmed histological or cytological diagnosis of bronchogenic carcinoma as per the WHO classification were included in the study. Staging of disease was conducted (at the time of their diagnosis) using AJCC (American Joint Committee on Cancer) criteria $7^{\text {th }}$ edition. Their demographic profile, clinical correlates, radiological profile and diagnostic details were studied. Benign tumors, malignant pleural disease and sarcomatoid tumors were excluded from this study.

The investigation methods include radiological assessment (postero-anterior and lateral view), fiberoptic bronchoscopy, supplemented by pleural fluid analysis and cytological examination of regional lymph nodes and metastatic deposits 
as appropriate. Descriptive statistics was used for describing demographic and clinical characteristics. Chi-square test was used to identify the significance of difference in proportions of the data.

\section{Results}

Table 1 depicts the demographic information of the study subjects stratified into

Table 1. Demographic characteristics of lung cancer patients.

\begin{tabular}{|c|c|c|c|c|}
\hline Feature & Strata & $\operatorname{SCC}(n=191)$ & $\mathrm{AC}(\mathrm{n}=879)$ & $\operatorname{SCLC}(\mathrm{n}=179)$ \\
\hline \multirow[t]{5}{*}{ Age (years) } & $<40$ & $4(2.09 \%)$ & $64(7.28 \%)$ & $7(3.91 \%)$ \\
\hline & $41-50$ & $10(5.24 \%)$ & $121(13.77 \%)$ & $16(8.94 \%)$ \\
\hline & $51-60$ & $61(31.94 \%)$ & $256(29.12 \%)$ & $61(34.08 \%)$ \\
\hline & $61-70$ & $75(39.27 \%)$ & $298(33.90 \%)$ & $62(34.64 \%)$ \\
\hline & $>70$ & $41(21.47 \%)$ & $140(15.93 \%)$ & $33(18.44 \%)$ \\
\hline \multirow[t]{2}{*}{ Sex } & Female & $26(13.61 \%)$ & $260(29.58 \%)$ & $30(16.76 \%)$ \\
\hline & Male & $165(86.39 \%)$ & $619(70.42 \%)$ & $149(83.24 \%)$ \\
\hline \multirow[t]{5}{*}{ Residence } & East & $4(2.09 \%)$ & $26(2.96 \%)$ & $6(3.35 \%)$ \\
\hline & North & & $4(0.46 \%)$ & $1(0.56 \%)$ \\
\hline & OS & $5(2.62 \%)$ & $17(1.93 \%)$ & $5(2.79 \%)$ \\
\hline & South & $179(93.71 \%)$ & $818(93.06 \%)$ & $166(92.74 \%)$ \\
\hline & West & $2(1.05 \%)$ & $10(1.14 \%)$ & $1(0.56 \%)$ \\
\hline \multirow[t]{4}{*}{ BMI } & $25-30$ & $18(9.42 \%)$ & $60(6.83 \%)$ & $16(8.94 \%)$ \\
\hline & $30-35$ & $3(1.57 \%)$ & $15(1.71 \%)$ & $3(1.68 \%)$ \\
\hline & $35-40$ & $1(0.52 \%)$ & $4(0.46 \%)$ & $2(1.12 \%)$ \\
\hline & $<25$ & $61(31.94 \%)$ & $193(21.96 \%)$ & $61(34.08 \%)$ \\
\hline \multirow[t]{4}{*}{ Method of diagnosis } & Biopsy & $29(15.18 \%)$ & $114(12.97 \%)$ & $59(32.96 \%)$ \\
\hline & Bronchoscopy & $2(1.05 \%)$ & $4(0.46 \%)$ & $1(0.56 \%)$ \\
\hline & Cytology & $5(2.62 \%)$ & $21(2.39 \%)$ & $7(3.91 \%)$ \\
\hline & Histopathology & $159(83.25 \%)$ & $748(85.10 \%)$ & $112(62.57 \%)$ \\
\hline \multirow[t]{12}{*}{ Stage } & IA & & & $1(0.56 \%)$ \\
\hline & IB & & $2(0.23 \%)$ & \\
\hline & II & & $2(0.23 \%)$ & \\
\hline & IIA & & $2(0.23 \%)$ & \\
\hline & IIB & $1(0.52 \%)$ & $3(0.34 \%)$ & $1(0.56 \%)$ \\
\hline & III & $5(2.62 \%)$ & $18(2.05 \%)$ & $3(1.68 \%)$ \\
\hline & IIIA & $4(2.09 \%)$ & $7(0.80 \%)$ & $4(2.23 \%)$ \\
\hline & IIIB & $11(5.76 \%)$ & $13(1.48 \%)$ & $11(6.15 \%)$ \\
\hline & IIIC & & $2(0.23 \%)$ & \\
\hline & IV & $82(42.93 \%)$ & 397 (45.16\%) & $96(53.63 \%)$ \\
\hline & IVA & $3(1.57 \%)$ & $3(0.34 \%)$ & $2(1.12 \%)$ \\
\hline & IVB & $1(0.52 \%)$ & $5(0.57 \%)$ & $2(1.12 \%)$ \\
\hline
\end{tabular}


3 categories: Squamous cell (SCC), Adeno (AC) and Small cell carcinoma (SCLC). During the study period, AC was detected among $70.4 \%$ of patients, SCC among $15.3 \%$ and SCLC among $14.3 \%$ of lung cancer admissions. Male to female ratio was 2.95:1. It was found that the median age of lung cancer patients was 61 years. $>60 \%$ of lung cancer patients were from the $51-70$ year age-group. Although geographic information could not be retrieved for all the cases, many cases were reported from south India. Body mass index (BMI) assessment from the available data depicts $>20 \%$ subjects in the underweight category. $>60 \%$ patients were diagnosed histopathologically and $>40 \%$ of cases (across the three strata of cancers) were diagnosed during Stage IV of the disease.

It is evident from Table 2 that across the three cancer types, $>35 \%$ received palliative chemotherapy and $>20 \%$ received palliative chemotherapy + radiation among patients in Stage 3B to 4 strata (variable stratified as Stages 1 to $3 \mathrm{~A}$ and 3B to 4). The data does not show any difference for "sex" variable when assessing the stage at which cancer was diagnosed.

Table 3 shows that among all the cancer patients, 23.23\% were diabetic. There was no predisposition of "sex" variable for diabetes. However, a significant difference for the same was found among different age groups. There was no difference found for either of the sub-groups of lung cancer, whether SCC or AC or SCLC. There was no difference found for co-morbidities as well.

Figure 1 depicts the distribution of the 3 types of lung cancer over the 8 year period. It shows a year-by-year profound decline in the number of diagnosed AC cases whereas the decline was marginal for SCLC and SCC.

Figure 2 depicts the age distribution of patients among the 3 types of lung

Table 2. Stage-wise treatment of patients.

\begin{tabular}{|c|c|c|c|c|c|c|c|}
\hline \multirow[b]{2}{*}{ Feature } & \multirow[b]{2}{*}{ Strata } & \multicolumn{2}{|c|}{ SCC } & \multicolumn{2}{|c|}{$\mathrm{AC}$} & \multicolumn{2}{|c|}{ SCLC } \\
\hline & & $1 \_3 A(\mathrm{n}=9)$ & $3 B \_4(\mathrm{n}=97)$ & $1 \_3 A(\mathrm{n}=34)$ & $3 B \_4(\mathrm{n}=417)$ & $1 \_3 A(\mathrm{n}=9)$ & $3 B \_4(\mathrm{n}=111)$ \\
\hline \multirow[t]{11}{*}{ Treatment } & Adjuvant chemotherapy & $1(11.11 \%)$ & $1(1.03 \%)$ & $2(5.88 \%)$ & $1(0.24 \%)$ & $2(22.22 \%)$ & \\
\hline & Chemo + Radiotherapy & & & $1(2.94 \%)$ & $3(0.72 \%)$ & & $4(3.60 \%)$ \\
\hline & Concurrent chemo-radiotherapy & & $3(3.09 \%)$ & $9(26.47 \%)$ & $3(0.72 \%)$ & $4(44.44 \%)$ & $5(4.50 \%)$ \\
\hline & Definitive Radiotherapy & $2(22.22 \%)$ & & $5(14.71 \%)$ & $11(2.64 \%)$ & & $3(2.70 \%)$ \\
\hline & Neoadjuvant Chemo + Radiation & & & & & $1(11.11 \%)$ & \\
\hline & Neoadjuvant chemotherapy & $4(44.44 \%)$ & $9(9.28 \%)$ & $5(14.71 \%)$ & $18(4.32 \%)$ & & \\
\hline & Palliative Chemo + Radiation & & $21(21.65 \%)$ & $1(2.94 \%)$ & $88(21.10 \%)$ & & $30(27.03 \%)$ \\
\hline & Palliative chemotherapy & $2(22.22 \%)$ & $36(37.11 \%)$ & $4(11.76 \%)$ & $168(40.29 \%)$ & & $43(38.74 \%)$ \\
\hline & Palliative radiotherapy & & $19(19.59 \%)$ & & $71(17.03 \%)$ & & $13(11.71 \%)$ \\
\hline & Supportive care & & $5(5.15 \%)$ & $1(2.94 \%)$ & $37(8.87 \%)$ & & $11(9.91 \%)$ \\
\hline & Surgery & & & $1(2.94 \%)$ & $7(1.68 \%)$ & & \\
\hline \multirow[t]{2}{*}{$\begin{array}{c}\text { Sex-wise } \\
\text { staging of cancer }\end{array}$} & Female & $2(22.22 \%)$ & $13(13.40 \%)$ & $10(29.41 \%)$ & $121(29.02 \%)$ & $1(11.11 \%)$ & $20(18.02 \%)$ \\
\hline & Male & $7(77.78 \%)$ & $84(86.60 \%)$ & 24 (70.59\%) & 295 (70.74\%) & $8(88.89 \%)$ & 91 (81.98\%) \\
\hline
\end{tabular}


Table 3. Characteristics of lung cancer patients by baseline diabetes status.

\begin{tabular}{|c|c|c|c|c|c|}
\hline SI. No. & Strata & Totaln (\%) & $D M \mathrm{n}(\%)$ & Non-DMn (\%) & $\mathrm{p}$ value \\
\hline \multirow[t]{2}{*}{1} & Cancer patients & 1248 & $290(23.23)$ & $958(76.77)$ & \\
\hline & & & Sex & & \\
\hline \multirow[t]{5}{*}{2} & Female & $316(25.32)$ & $74(25.52)$ & $242(25.34)$ & 0.9961 \\
\hline & Male & $932(74.68)$ & $216(74.48)$ & $716(74.66)$ & \\
\hline & & & Age (yrs) & & \\
\hline & $<50$ & $195(15.63)$ & $20(6.9)$ & $175(18.25)$ & 0.0027 \\
\hline & $50-59$ & $343(27.48)$ & $80(27.59)$ & $263(27.42)$ & \\
\hline \multirow{4}{*}{3} & $60-69$ & $453(36.3)$ & $121(41.72)$ & $332(34.62)$ & \\
\hline & $70-79$ & $223(17.87)$ & $61(21)$ & $162(16.89)$ & \\
\hline & $>80$ & $34(2.72)$ & $8(2.76)$ & $26(2.71)$ & \\
\hline & & & Sub-type & & \\
\hline \multirow{3}{*}{4} & Adeno & $879(70.43)$ & $198(68.28)$ & $681(71.01)$ & 0.2756 \\
\hline & Small cell & $178(14.26)$ & 36 (12.41) & $142(14.81)$ & \\
\hline & Squamous cell & $191(15.3)$ & $56(19.31)$ & $135(14.08)$ & \\
\hline \multicolumn{6}{|c|}{ Other co-morbidities } \\
\hline & Respiratory & $37(2.88)$ & $10(3.43)$ & $19(1.98)$ & 0.9724 \\
\hline & Cardiovascular & $30(2.4)$ & $21(7.23)$ & $9(0.92)$ & \\
\hline \multirow[t]{4}{*}{5} & Neurological & $3(0.24)$ & $1(0.34)$ & $2(0.2)$ & \\
\hline & Tuberculosis & $7(0.56)$ & $1(0.34)$ & $6(0.62)$ & \\
\hline & Endocrine & $7(0.54)$ & $3(1.03)$ & $4(0.41)$ & \\
\hline & Nephrology & $2(0.16)$ & 0 & $2(0.2)$ & \\
\hline
\end{tabular}

\section{Frequency of Cancer}

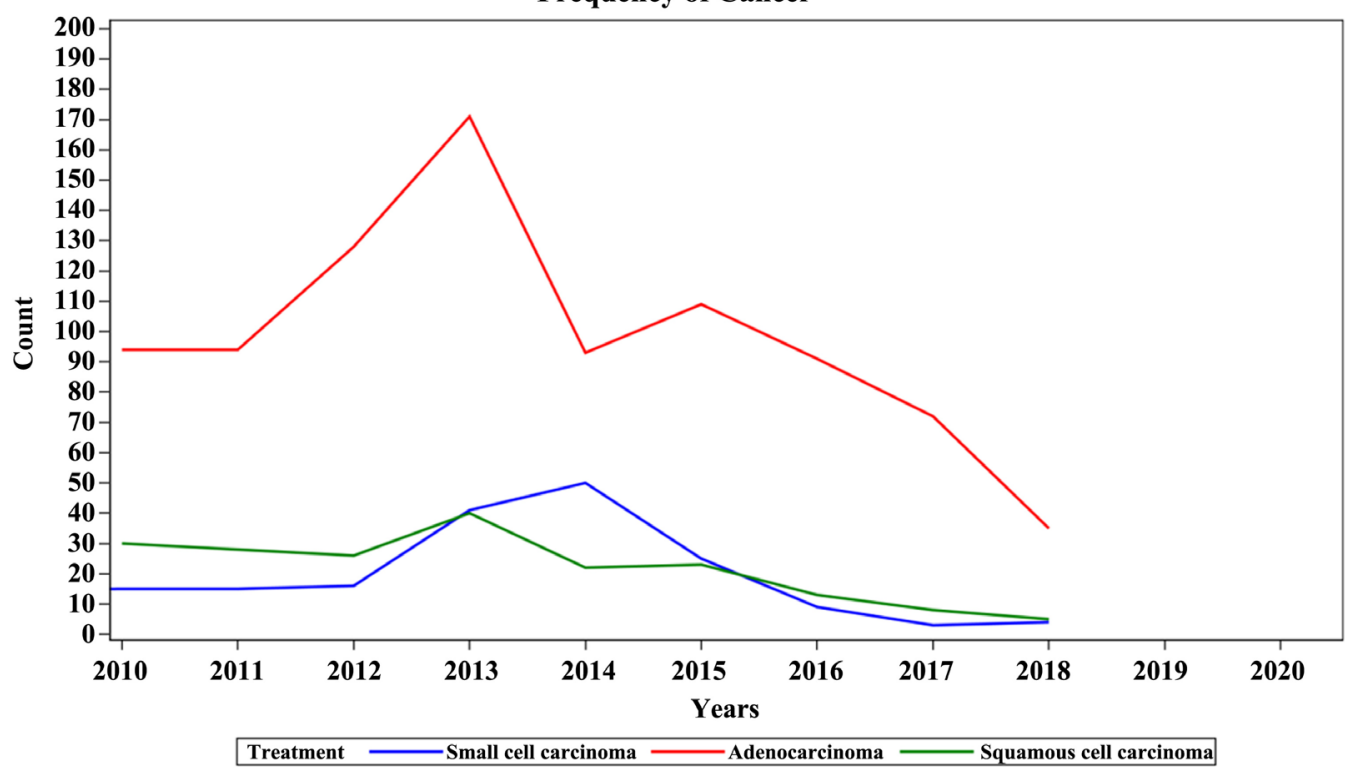

Figure 1. Year-wise plot of cancers. 


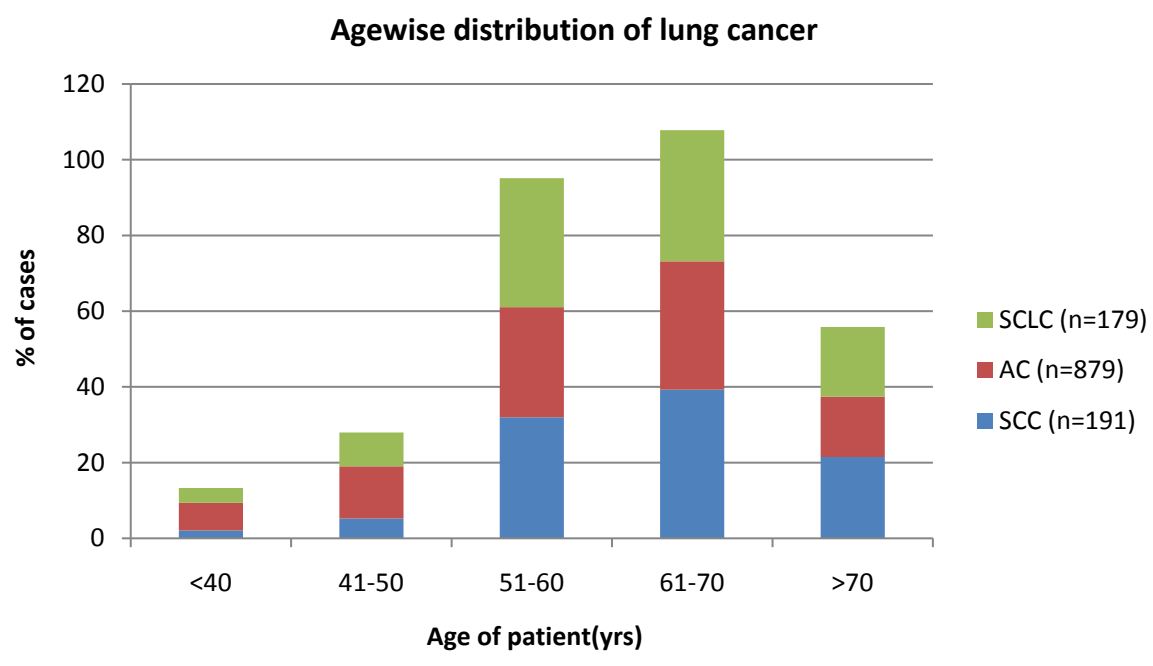

Figure 2. Age-wise plot of cancers.

cancer. The data show that a major proportion of all 3 cancer groups were found among patients in the age group of 51 to $70 \mathrm{yrs}$. In our study, $>60 \%$ of patients were in the $51-70$ year age group. Among those aged $<50 \mathrm{yrs}$, AC was the predominant type among all lung cancers.

Figure 3 depicts the sex distribution of patients among the 3 types of lung cancer. It shows that females comprise a larger proportion of AC ( 30\%) when compared to the other 2 cancer types. In our study, the male to female ratio was 2.95:1.

The genetic assessment of 1248 patients in our study shows EGFR (epidermal growth factor receptor) mutation in $42.8 \%(\mathrm{n}=534)$ patients as depicted in Table 4. EGFR mutation was the first biomarker identified as potential target for personalized treatments in lung cancer [9]. EGFR wild type was found in the remaining $57.2 \%(n=714)$ of cases. The most common EGFR mutation was exon 19 deletion (46.6\%) followed by exon 21 L858R (40\%) and exon 18 G719X (13.3\%).

\section{Discussion}

In our study, AC was the common histological sub-type (70.4\%) among the lung cancer patients, with SCC comprising $15.3 \%$ and SCLC diagnosed among $14.3 \%$ of patients. $>40 \%$ of patients were Stage IV at diagnosis. Cruz C.D et al. [10] in their analysis of SEER 2004-08 data in US report that SC comprise $15 \%$ of lung cancer cases. Of the remaining $85 \%$ of cases accounted by NSCLC, AC account for $38.5 \%$, SCC account for $20 \%$ and large cell carcinoma account for $2.9 \%$. This study reports a dismal 5 -year survival rate of $3.6 \%$ for patients with distant metastasis as compared with $52 \%$ for localized disease, which reiterates the need for screening of early-stage cancers.

Malik P.S [8] et al. in their study at AIIMS found 85.3\% NSCLC and 14.7\% SCLC cases, with $>55 \%$ cases being diagnosed at Stage IV or extensive disease. Dey A. [11] et al. found that $35.1 \%$ of their study subjects were SCC, $30.8 \%$ were 
Table 4. EGFR mutation among the study subjects.

\begin{tabular}{ccccc}
\hline Sl. No. & Type of mutation & Proportion & & \\
\hline & & & Type of EGFR mutation & Proportion \\
1 & EGFR mutation & $42.8 \%$ & exon 19 deletion & $46.6 \%$ \\
& & & exon 21 L858R & $40 \%$ \\
2 & EGFR wild type & $57.2 \%$ & exon 18 G719X & $13.3 \%$ \\
\hline
\end{tabular}

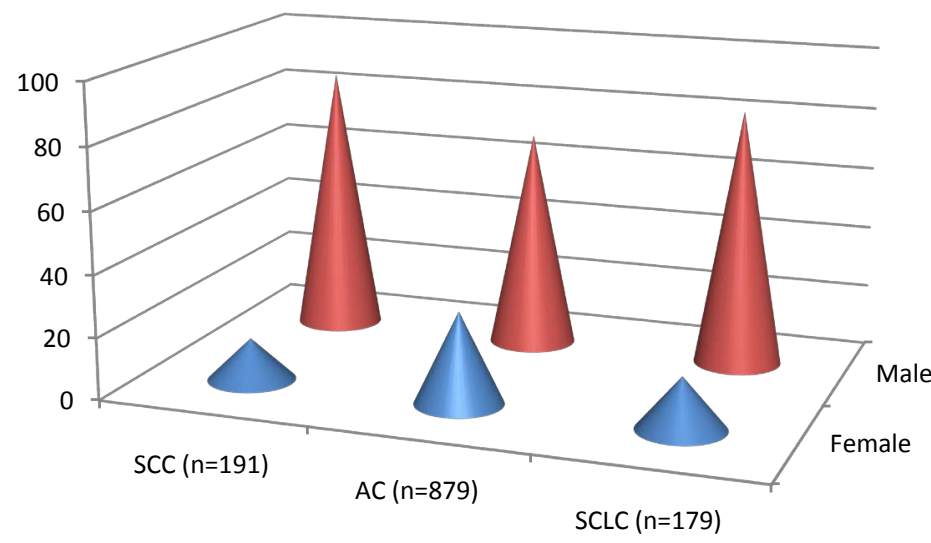

Female

Male

Figure 3. Sex-wise plot of cancers.

AC and 16.5\% comprised SCLC. SCC was the predominant type among males (35.99\%) and AC among females (40.68\%). It is possible that female smokers tend to use fewer cigarettes per day and inhale less intensely than males. Jindal S.K [6] found that SCC (34.3\%) were the most common histological cell type among 794 lung cancer cases in their study, followed by SCLC (20.3\%) and AC (25.9\%). Rawat et al. [5] report that SCC (44.83\%) outnumbered AC (19.7\%) and SLCC (16.75\%) in their study. Murali A.N et al. [12] report that $91 \%$ patients had NSCLC in their study, among which AC comprised $56.3 \%$ and SCC were $17.7 \%$.

The higher incidence of AC in our study cohort indicates a shift from the earlier predominance of SCC, which reflects changes in smoking practices and the current use of filter cigarettes which promotes deeper inhalation. Mitsudomi T [13] report the odds ratio (OR) for AC among smokers as 1.9 for males and 1.3 for females. We could infer that the contribution of smoking to AC is lower, especially among females. This study [13] also reports the OR for smoking in male/female patients as 21.4/12.1 and 18.1/9.7 respectively. These data indicate that it is rare to see SC or SCC in never-smokers. Cruz C.D et al. [10] report that $>80 \%$ of lung cancers occur in individuals exposed to tobacco, however $<20 \%$ of smokers develop lung cancer. The process of carcinogenesis is associated with individual susceptibility, and the influence of other environmental factors and genetic predisposition. The authors [10] also report that in Asian Countries such as Hong Kong, Singapore and Japan, lung cancer among never 
smokers is diagnosed at an earlier age than in smokers. Similar findings have not been reproduced in United States or Europe. The investigation threshold among symptomatic never smokers is higher leading to diagnosis at late stage. The survival rate for never smokers was better than for smokers, independent of stage of disease, treatment received and presence of co-morbidities. The late stage of diagnosis in our study is comparable with other Indian studies, as it indicates delay in seeking treatment. The decline in the number of cases across the 3 cancer groups over the study period could be because other centers in Bangalore city have initiated therapeutic management of lung cancers.

In Jindal S.K's [6] study, $40.2 \%$ of patients were $<50$ years of age. However, in our study $>60 \%$ of patients were in the $51-70$ year age group. Cruz C.D et al. [10] report that for the period 2004 to 2008, the Surveillance, Epidemiology and End Results (SEER) data show the median age at diagnosis for cancer of lung and bronchus as 71 years. No cases were reported for those aged $<20$ years, $0.2 \%$ of cases were in the age range of 20 to 34 years, $1.5 \%$ between 35 to 44 years, $20.9 \%$ between 55 to 64 years, $31.1 \%$ between 65 to 74 years, $29 \%$ between 75 to 84 years and $8.3 \%$ were $>85$ years old.

In our study, the male to female ratio was $2.95: 1$ which could be compared with Jindal S.K's [6] study which report the ratio as 4.5:1. Evidence indicates that woman had a 1.5 fold higher relative risk of lung cancer than men despite all odds [11]. Cheng et al. [14] in their global analysis of lung cancer epidemiology, report that the incidence of SCC is higher in Countries such as Belarus, India, Netherlands and Russia. Generally among males, the incidence of AC was higher than that for SCC (ratio > 1). This pattern was more evident among females, with ratio $>5$ reported from China, Japan and Saudi Arabia. This could be attributed to secondhand smoke and cooking related fumes. Cruz C.D et al. [10] report the findings from the American Cancer Society Cancer Prevention study II, where-in 1 to 2 million subjects were followed during the period 1982 to 1988. This study reported an overall risk of 11.94 and 22.36 for lung cancer among female and male smokers respectively, after accounting for intensity of smoking. This could be because of gender-related difference in nicotine metabolism, detoxification of lung carcinogens and other hormonal factors.

Our study reports that females comprised a larger proportion of AC ( 30\%) when compared to the other 2 cancer types. Also, $23.23 \%$ of all lung cancer patients were diabetic. A significant difference for the same was found among different age groups. Epidemiological studies provide evidence that diabetes and prediabetes are associated with increased risk of all cancers [15]. Karlin N.J et al. [16] in their matched case-control study found a five year overall survival for lung cancer patients with and without diabetes mellitus was $20 \%$ vs $29 \%$ ( $p=$ 0.12). The authors [16] conclude that neither diabetes adversely impacts lung cancer survival, nor does lung cancer affect the glycemic control. The progression of diabetes to cancer could be due to hyperinsulinemia, hyperglycemia and inflammation. Hyperinsulinemia through IGF-1 (Insulin like growth factor) possess mitogenic and antiapoptotic activities, which could play vital role in 
triggering cancer initiation [15]. Increased oxidative stress in diabetes is responsible for DNA damage, mutational change in oncogenes and eventually cancer.

In our study, EGFR mutations were reported among $42.8 \%$ of patients with a large proportion comprising deletion of exon 19, and mutation of exon 21 and 18. EGFR positive lung cancer is more common among non-smokers, AC type, women, young adults and asian ethnicity [13]. Werutsky G [17] et al. report a $33.07 \%$ median global prevalence of EGFR mutation (IQR 19.9\% - 47.52\%). The most common were $54.55 \%$ (IQR $45.45 \%$ - 67.5\%) in exon $19 \%$ and $36.36 \%$ (IQR 28.57\% - 47.06\%) in exon 21. This study [17] also reports a significantly higher median prevalence of EGFR mutation in Asian population (India, China, Japan and Taiwan in China) (42.7\%; p < 0.001), women (47.7\%: p < 0.001), non-smokers (53.6\%; p < 0.001) and AC (39.7\%; $\mathrm{p}<0.001)$. These results could be compared with Murali et al.'s [12] study which reports EGFR mutation among $62 \%$ of patients, with common sites being exon 19 and 22. The IPASS (First Line IRESSA versus Carboplatin/Paclitaxel in Asia) study also reported EGFR mutation among 59.7\% of AC type, with predominant exon 19 and 22 mutations. Singal G et al. [7] in their study to assess whether combining electronic health record (EHR) derived clinical data with clinical genomic profiling (CGP) among 4064 patients with NSCLC, found an alteration among a total $21.4 \%$ of patients in EGFR, ALK or ROS1 (17.2\%, 3.1\%, 1\% respectively). Genomic correlation with clinical outcomes will enable the generation of novel hypothesis, in particular related to immunotherapy. Analysis of such genomic data will enable interpreting the results of clinical trials and preventing carcinogenesis [18].

Molecular alterations such as mutation or gene amplification have the transforming/driver capability to fuel the growth of cancer cells. The ability of cancer cells to proliferate is presumed to be facilitated by networks of signaling pathways which evade cell cycle checks, thus inhibiting apoptosis, invading and generating distant metastatic deposits. In lung cancer, there is an increased likelihood of positive driver gene alterations. Epidermal growth factor receptor (EGFR) and KRAS activating mutations are the most common genetic alterations in AC. Liu et al. [19] in their study on young adults with lung cancer found that $45 \%$ of the 82 patients had AC and $49.21 \%$ had late-stage (Stage IV) disease at diagnosis. Among the 18 patients for whom EGFR and anaplastic lymphoma kinase (ALK) status was determined, 10 had sensitive EGFR mutations and 5 had ALK rearrangement. It is speculated from studies [19] on NSCLC among those aged $\leq 40$ years that frequent genomic alterations occur in this subset. We need to delineate genetic/molecular contributions as marker panels for risk and prognosis of lung cancer, which in-turn will address their application as screening tools in the clinical setting [20]. Assessment of gene mutation should be conducted on all lung cancer patients which in-turn could enable individualized targeted therapy.

In our country, lung cancer epidemiology is a reflection of the impact of industrialization and smoking trends in the community. Among many countries, 
AC has surpassed SCC as the most common histologic variant of lung cancer. This could be attributable to the changed smoking pattern and increased incidence of lung cancer among women and non-smokers. Certain evidence suggests that when compared to Caucasians, east Asians are more susceptible to smoking unrelated lung cancer but less susceptible to smoking related ones [13]. Racial differences have been studied by Cheng et al. [14] who report that african American males have a higher incidence of both AC and SCC than do white males given the same amount of smoking exposure. The authors [14] found that lung cancer was more likely to develop in asian americans than whites, given the similar levels of smoking. Various epidemiological factors such as sex, age and ethnicity of the patient influence the incidence of molecular alteration of a given driver oncogene.

The various treatment options available for the management of lung cancer include surgery, radiotherapy and chemotherapy. However, since most of the cases present in an advanced stage and radiation being a localized form of therapy, chemotherapy plays an important role in the management of lung cancer. Surgery has a limited role in SCLC [3]. Although the response of SCLC to systemic treatment is good, they are known to cause re-growth leading to relapse and high mortality. Singh N et al. [21] in their study on treatment pattern for lung cancer in resource constrained settings, report that cost of therapy, lack of medical insurance and frequency of visits as important determinants of treatment regimen. It is known that EGFR positive lung cancers have appropriate immune systems, as a result of which immunotherapy is rendered ineffective [9]. Apart from the traditional end points such as survival and response rates, treatment efficacy should assess the patient's quality of life, toxicity profile and utilization of institutional care.

Dikshit $\mathrm{R}$ et al. [22] in their million death study opine that data for the National Cancer Registry programme were primarily obtained from the urban cancer registries, and were not representative of the rural areas where most Indians habituate. Lin H.T et al. [23] estimated the overall survival rates of lung cancer by utilizing the Charlson Comorbidity index (CCI). In their study [23], it was found that lung cancer patients with CCI score $\leq 1$ ( $28.7 \%$ of total cases) had a better 5 -year survival rate $(24.5 \%$ vs $14.2 \%)$ compared to those with a score $\geq 2$ (71.3\% of total cases). Cardwell et al. [24] in their population-based lung cancer cohort, report a weak inverse association between statin usage and time to lung cancer-specific death ( $11 \%$ reduction, $\mathrm{p}=0.09)$.

\section{Conclusion}

It is essential to assess the clinic-demographic profile of lung cancer cases given the high fatality and the small variability in survival across geographic regions. Recent advances in diagnostic methods have enabled the detection of both gross as well as molecular changes in cancer tissues, and therapeutic advances have led to effective interventions. Research evidence on the prevalence of EGFR muta- 
tions among lung cancer cases will have an impact on designing future randomized clinical trials and public health policies. Beyond analysis of the clinical spectrum, follow-up of patients is essential to study the overall and progression free survival. Due to considerable heterogeneity of lung cancer patients, a multi-center study should be conducted to identify the properties. However, the outcome of lung cancer patients can be considerably impacted by addressing risk factors through preventive measures implemented in the community. Among high risk population, screening for lung cancer with low-dose computed tomography has been found to significantly reduce mortality. Certain non-invasive and cost-effective diagnostic techniques such as breath volatile organic compounds (VOC) need to be further validated for their role in the early detection of lung cancer.

\section{Limitations}

- We could not analyze the most common symptoms, site of metastasis and radiological presentation of the disease,

- Overall and progression free survival following treatment was not computed in our study,

- Data on immunohistochemistry (IHC) of cell block with markers such as synaptophysin and chromogranin along with Ki-67, could not be elicited,

- We could not retrieve the Eastern cooperative oncology group (ECOG) performance status (PS) scoring of patients.

\section{Acknowledgements}

1) Dr. Mithua Ghosh: Strand Life Sciences lab, HCG, Bangalore.

2) Dr. Veena R: Strand Life Sciences lab, HCG, Bangalore.

3) Amruth Anshuram: Medical Records Department, HCG, Bangalore.

4) Avinash Sevankar: Statistician, Biocon, Bangalore.

\section{Source of Funding}

None.

\section{Conflicts of Interest}

The authors declare no conflicts of interest regarding the publication of this paper.

\section{References}

[1] Lung Cancer Incidence by Sex, Age-Standardized Rate (World) per 100,000, 2018. https://canceratlas.cancer.org/the-burden/lung-cancer/

[2] Noronha, V., Pinninti, R., Patil, V.M., Joshi, A. and Prabhash, K. (2016) Lung Cancer in the Indian Subcontinent. South Asian Journal of Cancer, 5, 95-103. https://doi.org/10.4103/2278-330X.187571

[3] Ganguly, S., Biswas, B., Bhattacharjee, S., et al. (2020) Clinicopathological Characte- 
ristics and Treatment Outcome in Small Cell Lung Cancer: A Single Institutional Experience from India. Lung India, 37, 134-139.

https://doi.org/10.4103/lungindia.lungindia $370 \quad 19$

[4] Behera, D. and Balamugesh, T. (2004) Lung Cancer in India. The Indian Journal of Chest Diseases and Allied Sciences, 46, 269-281.

[5] Rawat, J., Sindhwani, G., Gaur, D., Dua, R. and Saini, S. (2009) Clinico-Pathological Profile of Lung Cancer in Uttarakhand. Lung India, 26, 74-76.

https://doi.org/10.4103/0970-2113.53229

[6] Jindal, S.K. and Behera, D. (1990) Clinical Spectrum of Primary Lung Cancer-Review of Chandigarh Experience of 10 Years. Lung India, 8, 94-98.

[7] Singal, G., Miller, P.G., Agarwala, V., et al. (2019) Association of Patient Characteristics and Tumor Genomics With Clinical Outcomes among Patients with Non-Small Cell Lung Cancer Using a Clinicogenomic Database. JAMA, 321, 1391-1399. https://doi.org/10.1001/jama.2019.3241

[8] Malik, P.S., Sharma, M.C., Mohanti, B.K., et al. (2013) Clinico-Pathological Profile of Lung Cancer at AIIMS: A Changing Paradigm in India. Asian Pacific Journal of Cancer Prevention, 14, 489-494. https://doi.org/10.7314/APJCP.2013.14.1.489

[9] EGFR-Positive Lung Cancer. https://lcfamerica.org/lung-cancer-info/types-lung-cancer/egfr-mutation/

[10] Cruz, C.D., Tanoue, L.T. and Matthay, R.A. (2011) Lung Cancer: Epidemiology, Etiology and Prevention. Clinics in Chest Medicine, 32, 605-644. https://doi.org/10.1016/j.ccm.2011.09.001

[11] Dey, A., Biswas, D., Saha, S.K., Kundu, S., Kundu, S. and Sengupta, A. (2012) Comparison Study of Clinicoradiological Profile of Primary Lung Cancer Cases: An Eastern India Experience. Indian Journal of Cancer, 49, 89-95. https://doi.org/10.4103/0019-509X.98930

[12] Mural, A.N., Radhakrishnan, V., Ganesan, T.S., et al. (2017) Outcomes in Lung Cancer: 9-Year Experience from a Tertiary Cancer Center in India. Journal of Clinical Oncology, 3, 459-468. https://doi.org/10.1200/JGO.2016.006676

[13] Mitsudomi, T. (2014) Molecular Epidemiology of Lung Cancer and Geographic Variations with Special Reference to EGFR Mutations. Translational Lung Cancer Research, 3, 205-211.

[14] Cheng, T.D., Cramb, S.M., Baade, P.D., et al. (2016) The International Epidemiology of Lung Cancer: Latest Trends, Disparities and Tumor Characteristics. Journal of Thoracic Oncology, 11, 1653-1671. https://doi.org/10.1016/j.jtho.2016.05.021

[15] Abudawood, M. (2019) Diabetes and Cancer: A Comprehensive Review. Journal of Research in Medical Sciences, 24, 94. https://doi.org/10.4103/jrms.JRMS 24219

[16] Karlin, N.J., Amin, S.B., Buras, M.R., et al. (2018) Patient Outcomes from Lung Cancer and Diabetes Mellitus: A Matched Case-Control Study. Future Science OA, 4, FSO248. https://doi.org/10.4155/fsoa-2017-0081

[17] Werutsky, G., Debiasi, M., Sampaio, F.H., et al. (2016) Updated Analysis of Global Epidemiology of EGFR Mutation in Advanced Non-Small Cell Lung Cancer. Journal of Thoracic Oncology, 11, S184-S185. https://doi.org/10.1016/j.jtho.2016.08.030

[18] Izumi, M., Suzumura, T., Ogawa, K., et al. (2019) Differences in Molecular Epidemiology of Lung Cancer among Ethnicities (Asian vs. Caucasian). Journal of Thoracic Disease, 12, No. 7.

[19] Liu, B., Quan, X., Xu, C., et al. (2019) Lung Cancer in Young Adults Aged 35 Years or Younger: A Full-Scale Analysis and Review. Journal of Cancer, 10, 3553-3559. 
https://doi.org/10.7150/jca.27490

[20] Schwartz, A.G., Prysak, G.M., Bock, C.H. and Cote, M.L. (2007) The Molecular Epidemiology of Lung Cancer. Carcinogenesis, 28, 507-518.

https://doi.org/10.1093/carcin/bgl253

[21] Singh, N., Aggarwal, A.N. and Behera, D. (2012) Management of Advanced Lung Cancer in Resource-Constrained Settings: A Perspective from India. Expert Review of Anticancer Therapy, 12, 1479-1495. https://doi.org/10.1586/era.12.119

[22] Dikshit, R., Gupta, P.C., Ramasundarahettige, C., et al. (2012) Cancer Mortality in India: A Nationally Representative Survey. The Lancet, 379, 1807-1816. https://doi.org/10.1016/S0140-6736(12)60358-4

[23] Lin, H.T., Liu, F.C., Wu, C.Y., et al. (2019) Epidemiology and Survival Outcomes of Lung Cancer: A Population-Based Study. BioMed Research International, 2019, Article ID: 8148156. https://doi.org/10.1155/2019/8148156

[24] Cardwell, C.R., Mc Menamin, Ú., Hughes, C.M. and Murray, L.J. (2015) Statin Use and Survival from Lung Cancer: A Population-Based Cohort Study. Cancer Epidemiology, Biomarkers \& Prevention, 24, 833-841.

https://doi.org/10.1158/1055-9965.EPI-15-0052 